Canadian

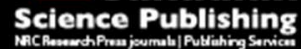

Canadian Journal of Microbiology Revue canadienne de de microbiologie

\title{
Reliability of Haemophilus influenzae biofilm measurement via static method and determinants of in vitro biofilm production.
}

\begin{tabular}{|r|l|}
\hline Journal: & Canadian Journal of Microbiology \\
\hline Manuscript ID & cjm-2016-0228.R1 \\
\hline Manuscript Type: & Article \\
\hline Date Submitted by the Author: & 11 -Jun-2016 \\
\hline Complete List of Authors: & $\begin{array}{l}\text { Obaid, Najla; University of Tasmania, School of Medicine } \\
\text { Tristram, Stephen; University of Tasmania, School of Health Sciences } \\
\text { Narkowicz, Christian; University of Tasmania School of Medicine, } \\
\text { Jacobson, Glenn; University of Tasmania, School of Medicine }\end{array}$ \\
\hline Keyword: & $\begin{array}{l}\text { Biofilm formation, NTHi, reproducibility, bacterial isolate storage, Microtitre } \\
\text { plate assay }\end{array}$ \\
\hline
\end{tabular}

\section{SCHOLARONE ${ }^{m}$ \\ Manuscripts}




\title{
Reliability of Haemophilus influenzae biofilm measurement via static method and determinants of in vitro biofilm production.
}

\author{
Najla A. Obaid ${ }^{1}$, Stephen Tristram², Christian K Narkowicz ${ }^{1}$, Glenn A Jacobson ${ }^{1}$. \\ ${ }^{1 .}$ Pharmacy division, School of Medicine, Hobart, University of Tasmania, Australia. \\ ${ }^{2}$ School of Health Sciences, University of Tasmania, Launceston, Tasmania, \\ Australia $^{2}$.
}

Corresponding author.

Najla Obaid

Email.najla.obaid@utas.edu.au

ph. +61362262206 Fax +61362262870

Private Bag 26, Hobart, 7005, TAS,

Australia.

\section{Declaration}

This work has not been submitted to any other journal or any other type of publication. Some parts of this project were presented as a poster at the Australian Society for Microbiology Annual Scientific Meeting on July 2013. 
Reliability of Haemophilus influenzae biofilm measurement via static method.

\begin{abstract}
Microtitre plate (MTP) assays used to measure biofilm are generally poorly described with regard to precision. This study investigated the precision of an MTP assay for biofilm production by Nontypeable Haemophilus influenzae (NTHi) and the effects of frozen storage and inoculation technique on biofilm production. The density of bacterial final growth (FG) was determined by absorbance after 18-20 hours incubation and biofilm production $(\mathrm{BF})$ was then measured by absorbance after crystal violet staining. Biofilm formation was categorised as high and low for each strain. For the high biofilm producing strains of NTHi, inter-day reproducibility of NTHi biofilm formation measured using the MTP assay was excellent and met the acceptance criteria but higher variability was observed in low biofilm producers. Method of inoculum preparation was a determinant of biofilm formation with inoculum prepared directly from solid media showing increased biofilm production for at least one of the high producing strains. In general, storage of NTHi cultures at $-80^{\circ} \mathrm{C}$ for up to 48 weeks did not have any major effect on their ability to produce biofilm.
\end{abstract}

\title{
Key words
}

Biofilm formation, NTHi, reproducibility, bacterial isolate storage, microtitre plate assay. 


\section{Introduction}

When planktonic bacteria aggregate and become part of a protected cellular community, they are termed a biofilm. Biofilm formation is a staged and circular process that is an inherent behavioural mechanism of some bacterial species (Costerton et al. 1999, Hall-Stoodley and Stoodley, 2009).

Nontypeable Haemophilus influenzae bacteria (NTHi) are obligate commensals of the human upper respiratory tract from where they frequently act as opportunistic pathogens. Biofilm production by NTHi may be responsible for chronicity and recurrence of some acute infections, such as otitis media $(\mathrm{OM})$ and exacerbation of chronic obstructive pulmonary disease (COPD), conditions that have provided most of the NTHi strains used for biofilm investigations (Hall-Stoodley and Stoodley, 2009, Murphy et al. 2004, Murphy and Kirkham, 2002, Swords, 2012, Moriyama et al. 2009).

In vitro assays of biofilms, based on microtitre plate (MTP) methods, have been developed over the past few decades (Christensen et al. 1985, Stepanović et al. 2000, Stepanović et al. 2007, Coenye and Nelis, 2010). The biofilm that adheres to the MTP is quantified by staining with crystal violet, a basic dye that binds to the biofilm matrix and attached cells. Although some workers consider biofilm production as measured in the static MTP assay as a poor reflection of the ability of a particular strain to form biofilm in vivo, closed system MTP models are still widely used and have included the development of proprietary systems (McBain, 2009). By comparison with various flow systems that have been developed (Goeres et al. 2005) 
static systems are simpler, require less specialised equipment, are more rapid and less expensive (Coenye and Nelis, 2010) with the potential for automation (Sandberg et al. 2008). The American Society for Testing and Materials recently approved the first standardised biofilm disinfectant efficacy test method, based on static culture of biofilm on peg lids that sit in MTP wells (Harrison et al., 2010, Parker et al., 2014). This technique has been applied to NTHi strains from acute OM cases (Takei et al. 2013) and CF and COPD isolates (Starner et al. 2006). However, a robust method for the quantitation of biofilm produced by NTHi is still lacking.

A lack of standardisation of inoculum, insufficient calculation methods and a lack of reporting of intra- and inter-day replicate variation of quantitative biofilm methods are typically found missing in key literature. The lack of standardised methods makes any meaningful comparison of different quantitative studies difficult. Studies have reported substantial variability in the ability of clinical isolates of NTHi to form biofilm (Murphy and Kirkham, 2002, Webster et al. 2006a), but inoculum was not standardised. Other studies reported variable NTHi biofilm production in vitro from different clinical isolates using standardised inoculum (Puig et al. 2014, Wu et al. 2014). Other work has described the use of a ratio termed the biofilm formation index (BFI). This is the ratio of biofilm produced by an NTHi isolate to the biofilm produced by a standard Haemophilus influenzae strain (Moriyama et al., 2009). This approach has merit but is limited by its reliance on the reproducible production of biofilm by the control, which cannot be relied upon, given the fastidious nature of NTHi (Daines et al. 2005). Determination of quantitative precision and reproducibility requires a number of replicates over different days, which is more readily achieved in a static system (Goeres et al. 2005). More broadly, due to the lack 
of a reliable reported standardised methodology there is still considerable uncertainty as to the clinical significance of biofilms in the pathogenesis of NTHi infections, however, we have previously reported that the quantity of NTHi biofilm production as measured using a MTP assay on 50 clinical isolates can be associated with certain clinical sites (Obaid et al. 2015).

The aim of this study was to develop and validate a reproducible and robust crystal violet MTP assay for the quantitative assessment of NTHi biofilm production using a small number of representative clinical isolates and standard strains, and to use this method to measure potential determinants of biofilm production, including isolate storage and inoculum preparation techniques.

\section{Methods}

\subsection{Strains and culture condition}

Two isolates of NTHi, comprising clinical isolates (A1 and A2 respectively) and two standard strains, ATCC 10211 and Rd KW20 (A3 and A4 respectively), as well as Pseudomonas aeruginosa PAO1 as a positive control, were used for biofilm evaluation. The strains were stored in glycerol at $-80^{\circ} \mathrm{C}$ and recovered by overnight culture followed by an additional overnight (16 h) passage prior to use in the assay. All cultures were carried out on chocolate agar (Oxoid, Adelaide, Australia) at $37^{\circ} \mathrm{C}$ under $5 \% \mathrm{CO}_{2}$. Additional NTHi clinical isolates were used for the investigation of storage, as described below. All clinical isolates were epidemiologically unrelated and were identified conclusively as $H$. influenzae using $16 \mathrm{~S}$ rRNA sequencing. 
Reliability of Haemophilus influenzae biofilm measurement via static method.

\subsection{Baseline biofilm assay}

Strains A1-A4 were preliminarily screened for their ability to form biofilm using an in vitro MTP assay adapted from published methods (Murphy and Kirkham, 2002, Webster et al., 2006b). One colony from the overnight passage culture was inoculated into $10 \mathrm{ml}$ pre-warmed brain heart infusion broth (Oxoid, Adelaide, Australia) supplemented with $40 \mu \mathrm{g} / \mathrm{ml} \mathrm{NAD}$ and haemin and 2\% v/v Vitox (Oxoid, Adelaide, Australia) (sBHI), and incubated for $22 \mathrm{~h}$. A $1.5 \mathrm{ml}$ aliquot of each broth was transferred into a plastic cuvette (Kartell, Milan, Italy) and the optical density was measured at $490 \mathrm{~nm}$ versus fresh sBHI. Based on this reading, each inoculum was standardised by dilution with sBHI to an optical density of $0.25-0.30\left(-10^{7} \mathrm{CFU} / \mathrm{ml}\right)$ which was confirmed by producing a growth curve of the counted colonies for NTHi against OD measurement. The final inoculum was prepared by adding $100 \mu 1$ of the standardised broth to $10.0 \mathrm{ml}$ of fresh sBHI (1:100). Diluted inoculum $(2.0 \mathrm{ml})$ was transferred to wells $(2.5 \mathrm{ml})$ of a 24 -well non-treated sterile, polystyrene microtitre plate (MTP) (Costar, Corning Incorporated, New York, USA). Each MTP contained 4 NTHi strains, a positive control (PAO1) and a negative control (sBHI with no inoculum) in quadruplicate wells with a single plate. Plates were incubated at $37^{\circ} \mathrm{C}$ under $5 \% \mathrm{CO}_{2}$ for $22 \mathrm{~h}$. After incubation, MTPs were cooled to room temperature and then covered with an adhesive film (Labtek, Brendale, Australia). The optical density of each well was read at $490 \mathrm{~nm}$ to give a value for final growth (FG).

MTPs were inverted to empty, and then washed with tap water (3x) and air dried at room temperature for $30 \mathrm{~min}$. Crystal violet solution $(0.1 \%, 2.0 \mathrm{ml})$ was added to each well and allowed to sit at room temperature for $15 \mathrm{~min}$. MTPs were inverted to empty, washed with water $(3 \mathrm{x})$ and air dried at room temperature. Ethanol $(95 \%, 2.0 \mathrm{ml})$ was 
added to each well to solubilise the crystal violet that had adhered to the biofilm. The optical density of each well was read at $570 \mathrm{~nm}$ to give a value for biofilm formation (BF).

In total, 13 MTP assays were performed (with strains A1-A4, plus positive and negative controls) over five days. On days one to four, two MTP assays were performed, and on day five, five MTP assays were performed. Unless otherwise indicated (e.g., for the determination of a particular analytical performance), the FG, $\mathrm{BF}$ and $\mathrm{BF} / \mathrm{FG}$ for each strain within a single MTP was taken as the mean of the individual values from the four individual wells. For management of outliers (such as contamination or no growth), an a priori protocol for exclusion was used whereby wells with a FG value more than \pm 2 standard deviations from the mean of each of the quadruplicate wells within a plate were excluded.

\subsection{Outcome measures}

Final growth (FG) was measured using turbidity of the bacterial suspension in the well as a measure of overall growth of biomass, however this can often be highly variable between strains. Biofilm formed inside the wells determined by crystal violet staining was defined as absolute biofilm $(\mathrm{BF})$. The ratio $(\mathrm{BF} / \mathrm{FG})$ was then calculated and defined as relative biofilm formation, normalising for bacterial growth.

\subsection{Precision and reproducibility}

Intra-day precision was assessed using the MTP results from day 5, and comparing the mean values $(\mathrm{BF}$ and $\mathrm{BF} / \mathrm{FG})$ for each strain from each of 5 MTPs $(\mathrm{n}=5)$. Interday precision was assessed using the MTP results from all 5 days, and comparing the 
Reliability of Haemophilus influenzae biofilm measurement via static method.

mean values $(\mathrm{BF}$ and $\mathrm{BF} / \mathrm{FG})$ for each strain from each of 13 MTPs $(\mathrm{n}=13)$. In order

to determine if strains could be reliably placed into categories based on biofilm production (e.g. high or low), the 13 means from the inter-day precision assay were compared, with an acceptance criteria of $\pm 20 \%$ RSD arbitrarily assigned.

\subsection{Effect of inoculum}

A non-standardised inoculum was used to evaluate the importance of inoculum standardization on biofilm quantitation. Incubations were performed in a similar manner to that described above but without adjustment of the inoculum to an optical density of $0.25-0.3$ prior to $1: 100$ dilution and incubation of all four strains.

To evaluate the importance of inoculum pre-culture in liquid broth, the inocula was prepared from a culture off chocolate agar solid media. The inocula was standardised to an optical density of $0.25-0.3$, diluted $1: 100$ and incubated and processed as previously described.

The effect of inoculum was assessed using two MTPs on a single day for each of the three inoculum types on five MTP assays on day five were performed comparing the variation across the plates of each day.

\subsection{Effect of isolate storage}

The effect of freezing on the ability of NTHi to form biofilm was investigated. Fresh NTHi clinical isolates (B1, B2, B3 and B4) were collected and evaluated for biofilm formation. These isolates had undergone less than four sub-cultures following initial isolation from clinical specimens. The MTP assay was performed within two days of 
initial isolation, with two MTP assays performed per strain each day, to generate a single mean value for $\mathrm{BF}$ from eight individual wells. The same analyses were performed after freezing in glycerol on ceramic beads and storage at $-80^{\circ} \mathrm{C}$ for two, four, eight, 12, 24 and 48 weeks.

\subsection{Statistical analysis}

For comparison of biofilm (BF) and relative biofilm formation (BF/FG) between strains and to examine the differences in biofilm production within strains, Student's t-test and one-way repeated measures ANOVA (IBM SPSS Statistics, V21.0) were used along with nonparametric tests where appropriate with $\mathrm{P}<0.05$ considered statistically significant. For comparison of the three different inoculum methods and classification of biofilm (BF) and relative biofilm (BF/FG) production (high and low), a general linear model (GLM) and univariate analysis of variance based on individual wells was used to produce a two-way repeated measures ANOVA (IBM SPSS Statistics, V21.0) with $\mathrm{P}<0.05$ considered statistically significant. 
Reliability of Haemophilus influenzae biofilm measurement via static method.

\section{Results}

\subsection{Baseline biofilm assay}

Biofilm formation (BF) over five different days for each of the tested strains are presented in Fig. 1. Each bar represents the mean BF on a particular day with consistency in the amount of biofilm produced between strains. It was evident that A1 and $\mathrm{PAO} 1$ produced a similar amount of biofilm $(\mathrm{BF})(\mathrm{P}>0.05)$. Strain A1 produced significantly more biofilm than strain A2 $(\mathrm{P}<0.01)$, strain A3 $(\mathrm{P}<0.01)$ and strain A4 $(\mathrm{P}<0.01)$. Strain $\mathrm{A} 2$ produced significantly more biofilm than $\mathrm{A} 3(\mathrm{P}<0.01)$ and $\mathrm{A} 4$ $(\mathrm{P}<0.01)$ and strains $\mathrm{A} 3$ and $\mathrm{A} 4$ produced a similar amount of biofilm $(\mathrm{P}>0.05)$. Discriminatory categories of biofilm formation were calculated as; high $(\geq 0.6)$ and low $(<0.6)$ producers $(\mathrm{P}<0.05)$.

Relative biofilm production (BF/FG ratio) data are presented in Fig. 2. Strain A1 gave a BF/FG ratio higher than all the other strains including PAO1 $(\mathrm{P}<0.01)$. The BF/FG ratios for all of NTHi strains gave similar relative results as for the BF data (P values were very similar). The percent relative standard deviation (\%RSD) was calculated for each of the five days using both $\mathrm{BF}$ and $\mathrm{BF} / \mathrm{FG}$ data which are presented in Table 1. There were no differences in \%RSD intra-day variability $(\mathrm{P}>0.05)$ or inter-day variability $(\mathrm{P}>0.05)$ between using $\mathrm{BF} / \mathrm{FG}$ ratio versus $\mathrm{BF}$ for any of the strains. Based on the a priori exclusion protocol (FG value more than \pm 2 standard deviations from the mean of each of the quadruplicate wells within a plate), four wells for strain A1, two wells for strain A2 and six wells in strain A3 were excluded over the course of experiments. 
Reliability of Haemophilus influenzae biofilm measurement via static method.

\subsection{Effects of inoculum preparation}

The effect of inoculum from standardisation, non-standardisation and solid inoculum are illustrated in Fig. 3. Strains A1 and A2 demonstrated no difference in BF between inoculation techniques $(\mathrm{P}>0.05)$. Strain $\mathrm{A} 3$ and $\mathrm{A} 4$ increased $\mathrm{BF}$ measurement with solid inoculum over the other inoculum techniques $(\mathrm{P}<0.05)$ (see Table 2) with biofilm formation doubling in strain A3 prepared from solid inoculum compared to inoculum prepared from standardised liquid inoculum $(\mathrm{P}<0.05)$. Relative biofilm production BF/FG in Fig. 4 gave similar results in terms of the biofilm level production that presented in the BF value Fig. 3 .

\subsection{Effect of isolate storage}

In Fig. 5, the results of the frozen storage in $-80^{\circ} \mathrm{C}$ experiments demonstrated that biofilm production was not affected in most cases and that biofilm production only changed in two strains (B2 and B3) after six months $(\mathrm{P}<0.05)$. There was some fluctuation of biofilm production (BF) for B2. 
Reliability of Haemophilus influenzae biofilm measurement via static method.

\section{Discussion}

\subsection{Baseline biofilm assay}

The ability to reliably and consistently discriminate between high and low biofilm producing NTHi strains was achieved with the MTP assay method. Of the NTHi strains that were examined, strain A1 was classified as a high biofilm producer, and strains A2, A3 and A4 were low biofilm producers. A1 produced similar amounts of biofilm (BF) as the control strain (PAO1). Strain A2, although categorised as a low biofilm producer, produced around $60 \%$ of the biofilm of strain A1 but significantly more than A3 and A4 which both produced only about $10 \%$ of A1's biofilm indicating difficulty of classification of low biofilm producers. The method met acceptance criteria of precision and repeatability ( $\pm 20 \%$ of $\% \mathrm{RSD})$ for biofilm in most of the strains measured by MTP assay.

H. influenzae strains ATCC 10211 (A3) and RdW20 (A4) were chosen in addition to unique local strains, because they are widely available and could act as reference isolates for others wishing to use or evaluate our method. ATCC 10211 was especially chosen because it is known to be particularly fastidious and is used by the Clinical Laboratory Standards Institute for quality control of susceptibility testing media (C.L.S.I, 2012). The four strains were also selected to cover a range of biofilm forming ability. In the context of this work, P. aeruginosa (PAO1) strain served as positive control as it has been extensively studied and confirmed as a high biofilm producer in MTP assays (Bjarnsholt et al., 2005, Hall-Stoodley and Stoodley, 2009). The reason for choosing $P$. aeroginosa (PAO1) strain was that there was no NTHi strain confirmed to be a robust high biofilm producer to serve as positive control. 
Relative biofilm production (BF/FG ratio) normalises biofilm production to the bacterial density in each well. The use of this ratio could correct for potential variations in bacterial growth in different wells. However, in the current study, the use of this ratio made no difference to the assay performance measures of intra-day and inter-day variability, compared with the use of BF alone. This indicates that bacterial growth was consistent in each well even for the known fastidious strain A3. For example, intra-day variability of final growth (FG) for strain A3 was 7.7\% (\%RSD) which gives a further indication of the reproducibility of the final growth. The extra step of measuring bacterial density in order to calculate this ratio cannot be justified on the basis of our results. However, there may be a benefit in using the ratio for measurement of biofilm production after treating the inoculum with antibacterial agents where variation in bacterial growth may have more experimental variability (Harrison et al., 2010).

The acceptance criteria of RSD of $\pm 20 \%$ variability were met only for BF of strains A1 and A2. Biofilm productions of strains (A3 and A4) were less reproducible and greater than the acceptance criteria. Inter-day variability tended to increase with A3 and $\mathrm{A} 4$, the low biofilm producers, but the correlation between BF and variability was weak. Inter-day precision of MTP biofilm measurements are not routinely described in the literature, but should be taken into account when determining the validity of biofilm quantitation using static methods. As a comparison, reproducibility of biofilm production (BF) by MTP assay for Staphylococcus aureus was reported as 35\%, greater than that shown here for NTHi strain A1 and A2 (Sandberg et al. 2008). 
Reliability of Haemophilus influenzae biofilm measurement via static method.

\subsection{Effects of inoculum preparation}

Examination of the final growth measurements shows that all strains grew to a similar cell density after full incubation, regardless of the type of initial inoculum preparation. On this basis, as standardisation of the inoculum by dilution to a specified $0.25-0.30$ OD 490 does not increase reproducibility, it is not shown to be necessary.

Solid growth inoculum made no difference for biofilm production, even after vigorously shaking the inoculum after inoculation from solid media, except for strains A3 and A4 Fig. 3. The effect of inoculum from chocolate agar was investigated because many studies do not define the source of cells for the preparation of their final inoculum. Results from solid media are not directly comparable with results of inoculum from liquid media. Stepanović et al. (2007) have suggested that adhesion to cell surfaces can be initiated in the first step of biofilm formation and potentially resulting in more biofilm formation from bacterial cells grown on solid medium than bacterial cells grown in liquid medium (Stepanović et al. 2007).

In this study, we chose to use 24 well, flat bottom untreated well MTPs for several reasons. Plates with 24 wells have been used for MTP assays in some previous studies for other organisms as reviewed by Coenye and Nelis (2010) and specifically for NTHi biofilm studies (Murphy and Kirkham, 2002, Webster et al. 2006a, Ünal et al. 2012). These plates have a larger absolute surface area than other types which were used in other published studies (Stepanović et al. 2007). The microtitre plates were specifically flat bottom polystyrene non-tissue culture treated wells. Non-treated wells were chosen, free of any interference from other factors that may enhance the 
adhesion of cells and initial biofilm formation. Tissue culture treated wells have been reported in some papers as an enhancer of biofilm formation (Stepanović et al. 2007, Murphy and Kirkham, 2002). Investigation of the effects of well size and coating types on biofilm production were not conducted in this current study due to the myriad of combinations possible, but clearly these factors should be taken into account when comparing across biofilm studies using MTP methods.

\subsection{Effect of isolate storage}

These fresh clinical isolates were chosen to cover a similar range of biofilm producing ability as the strains used in the earlier study. There appeared to be some strainspecific changes in biofilm production found after frozen storage of freshly cultured cells over 48 weeks, however, these changes were within the overall inter-day variability of the MTP assay. Frozen storage affected biofilm production of strain (B2) after 24 weeks in $-80^{\circ} \mathrm{C}$ Fig. 5. There were also some differences in biofilm production in strain B3 which were found after eight weeks of freezing compared to fresh, and after 48 weeks. Biofilm production is the result of a complex interaction between bacterial cells and the host surfaces and there is no information on whether the ability to form biofilms is retained when the bacterial cells are removed from the host and stored for long periods. This is an important consideration as in most studies, strains are stored prior to being tested. To date, there are no studies investigating the biofilm producing ability for NTHi isolates after short or long periods of freezing. Two studies have tested the consequence of freezing; $H$. influenzae planktonic strains were stored in different types of media and repeated viability tests were undertaken after thawing/freezing cycles (Saab et al. 2001, Votava and Strítecká, 2001). Viability of planktonic Haemophilus influenzae decreases after freezing for longer periods of 
Reliability of Haemophilus influenzae biofilm measurement via static method.

time (>48 weeks) (Saab et al. 2001). Therefore, we can determine from our study that the ability of NTHi strains to form biofilm may be affected by freezing over a 24 week period but appears to be strain-specific as this was not observed in all NTHi strains. 
Reliability of Haemophilus influenzae biofilm measurement via static method.

\section{Conclusion}

Different strains of NTHi consistently produced different levels of biofilm. The MTP assay met precision acceptance criteria for measuring biofilm production by NTHi strains. The only determinant of variability when measuring NTHi biofilm using the MTP assay is due to the particular NTHi strain. Standardisation of the inoculum (versus non-standardised) made no difference which could be a step reserved for more fastidious strains. The source of inoculum from culture media demonstrated less precision for at least one strain. Frozen storage duration showed no major effect on the biofilm formation as the biofilm production for some strain changed but within the inter-day variability of our MTP assay. The MTP assay is a valid approach to measuring NTHi biofilm and can detect some strain-specific differences in biofilm production, was well as the effect of inoculum techniques and frozen storage duration. 
Reliability of Haemophilus influenzae biofilm measurement via static method.

\section{References}

Bjarnsholt, T, Jensen, P. Ø, Burmølle, M, Hentzer, M, Haagensen, J, Hougen, H, P, Calum, H, Madsen, K, Moser, C, \& Molin, S, 2005. 'Pseudomonas aeruginosa tolerance to tobramycin, hydrogen peroxide and polymorphonuclear leukocytes is quorum-sensing dependent'. Microbiology, 151, 373-383.

C.L.S.I 2012. 'Methods for dilution antimicrobial susceptibility tests for bacteria that grow aerobically; approved standard, 9th edition M07-A9. Wayne, PA.

Christensen, G D, Simpson, W, Younger, J, Baddour, L, Barrett, F, Melton, D \& Beachey, E 1985. 'Adherence of coagulase-negative staphylococci to plastic tissue culture plates: a quantitative model for the adherence of staphylococci to medical devices'. Journal of Clinical Microbiology, 22, 996-1006.

Coenye, T \& Nelis, H J, 2010. 'In vitro and in vivo model systems to study microbial biofilm formation'. Journal of Microbiological Methods, 83, 89-105.

Costerton, J W, Stewart, P S, \& Greenberg, E P 1999. 'Bacterial biofilms: a common cause of persistent infections'. Science, 284, 1318-1322.

Daines, D A, Bothwell, M., Furrer, J, Unrath, W, Nelson, K, Jarisch, J, Melrose, N, Greiner, L, Apicella, M \& Smith, A L 2005. 'Haemophilus influenzae luxS mutants form a biofilm and have increased virulence'. Microbial Pathogenesis, 39, 87-96. 
Reliability of Haemophilus influenzae biofilm measurement via static method.

Goeres, D M, Loetterle, L R, Hamilton, M A, Murga, R, Kirby, D W \& Donlan, R M 2005. 'Statistical assessment of a laboratory method for growing biofilms'. Microbiology, 151, 757-762.

Hall-Stoodley, L \& Stoodley, P 2009. 'Evolving Concepts in Biofilm Infections'. Cellular Microbiology, 11, 1034-1043.

Harrison, J J, Stremick, C A, Turner, R J, Allan, N D, Olson, M E \& Ceri, H 2010. 'Microtiter susceptibility testing of microbes growing on peg lids: a miniaturized biofilm model for high-throughput screening'. Nature Protocols, 5, 1236-1254.

McBain, A 2009. 'Chapter 4: In vitro biofilm models: an overview.". Advanced Applied Microbiology, 69, 99-132.

Moriyama, S, Hotomi, M, Shimada, J, Billal, D S, Fujihara, K \& Yamanaka, N 2009. 'Formation of biofilm by Haemophilus influenzae isolated from pediatric intractable otitis media'. Auris Nasus Larynx, 36, 525-531.

Murphy, T F, Brauer, A L, Schiffmacher, A T \& Sethi, S 2004. 'Persistent colonization by Haemophilus influenzae in chronic obstructive pulmonary disease'. American Journal of Respiratory and Critical Care Medicine, 170, 266-272.

Murphy, T F \& Kirkham, C 2002. 'Biofilm formation by nontypeable Haemophilus influenzae: strain variability, outer membrane antigen expression and role of pili'. BMC microbiology, $2,7$. 
Reliability of Haemophilus influenzae biofilm measurement via static method.

Obaid, N A, Jacobson, G A \& Tristram, S 2015. ' Relationship between clinical site of isolation and ability to form biofilms in vitro in nontypeable Haemophilus influenzae.' Canadian Journal of Microbiology, 61(3), 243-245.

Parker, A, Walker, D, Goeres, D, Allan, N, Olson, M \& Omar, A 2014. 'Ruggedness and reproducibility of the MBEC biofilm disinfectant efficacy test'. Journal of Microbiological Methods, 102, 55-64.

Puig, C, Domenech, A, Garmendia, J, Langereis, J D, Mayer, P, Calatayud, L, Liñares, J, Ardanuy, C \& Marti, S 2014. 'Increased Biofilm Formation by Nontypeable Haemophilus influenzae Isolates from Patients with Invasive Disease or Otitis Media versus Strains Recovered from Cases of Respiratory Infections'. Applied and Environmental Microbiology, 80, 7088-7095.

Saab, O C, Castillo, M C, Ruiz Holgado, A P \& Nader, O M 2001. 'A comparative study of preservation and storage of haemophilus influenzae'. Memórias do Instituto Oswaldo Cruz, 96, 583-586.

Sandberg, M, Määttänen, A, Peltonen, J, Vuorela, P M \& Fallarero, A, 2008. 'Automating a 96-well microtitre plate model for Staphylococcus aureus biofilms: an approach to screening of natural antimicrobial compounds'. International Journal of Antimicrobial Agents, 32, 233-240. 
Starner, T D, Zhang, N, Kim, G, Apicella, M A \& Mccray Jr, P B, 2006. 'Haemophilus influenzae forms biofilms on airway epithelia: implications in cystic fibrosis'. American Journal of Respiratory and Critical Care Medicine, 174, 213.

Stepanović, S, Vuković, D, Dakić, I, Savić, B \& Švabić-Vlahović, M 2000. 'A modified microtiter-plate test for quantification of staphylococcal biofilm formation'. Journal of Microbiological Methods, 40, 175-179.

Stepanović, S, Vuković, D, Hola, V, Bonaventura, G D I, Djukić, S, Ćirković, I \& Ruzicka, F 2007. 'Quantification of biofilm in microtiter plates: overview of testing conditions and practical recommendations for assessment of biofilm production by staphylococci'. Apmis, 115, 891-899.

Swords, W E 2012. 'Nontypeable Haemophilus influenzae biofilms: role in chronic airway infections'. Frontiers in Cellular and Infection Microbiology, 2.

Takei, S, Hotomi, M \& Yamanaka, N 2013. 'Minimal biofilm eradication concentration of antimicrobial agents against nontypeable Haemophilus influenzae isolated from middle ear fluids of intractable acute otitis media'. Journal of Infection and Chemotherapy, 1-6.

Ünal, C M, Singh, B, Fleury, C, Singh, K, Chávez De Paz, L, Svensäter, G \& Riesbeck, K 2012. 'QseC controls biofilm formation of non-typeable Haemophilus influenzae in addition to an AI-2-dependent mechanism'. International Journal of Medical Microbiology. 
Reliability of Haemophilus influenzae biofilm measurement via static method.

Votava, M \& StríTecká, M 2001. 'Preservation of Haemophilus influenzae and Haemophilus parainfluenzae at- $70^{\circ} \mathrm{C}^{\prime}$. Cryobiology, 43, 85-87.

Webster, P, Wu, S, Gomez, G, Apicella, M, Plaut, A G \& Geme Iii, J W S 2006a. 'Distribution of bacterial proteins in biofilms formed by non-typeable Haemophilus influenzae'. Journal of Histochemistry \& Cytochemistry, 54, 829-842.

Webster, P, Wu, S, Gomez, G., Apicella, M., Plaut, A G. \& Geme Iii, J W S 2006b. 'Distribution of bacterial proteins in biofilms formed by non-typeable Haemophilus influenzae'. Journal of Histochemistry \& Cytochemistry, 54, 829.

Wu, S, Baum, M M, Kerwin, J, Guerrero, D, Webster, S, Schaudinn, C, Vandervelde, D \& Webster, P, 2014. 'Biofilm specific extracellular matrix proteins of nontypeable Haemophilus influenzae'. Pathogens and Disease. 72, 143-160. 


\section{Figures legends}

Figure 1. Biofilm production (BF) of the four NTHi strains (A1-A4) and Pseudomonas aeruginosa PAO1 positive control (PC) over five days. Error bars depict standard deviation.

Figure 2. Relative biofilm production $(\mathrm{BF} / \mathrm{FG})$ of the four NTHi strains (A1-A4) and Pseudomonas aeruginosa PAO1 positive control (PC) over five days. Error bars depict standard deviation.

Figure 3. Robustness comparison of the standardised method, solid growth and non-standardised inoculum for NTHi strains (A1-A4) and Pseudomonas aeruginosa PAO1 positive control (PC) for 8 wells over 13 plates. Error bars depict standard deviation and with statistical significance $\mathrm{P}<0.05^{*}$.PC represents the positive control Pseudomonas aeruginosa PAO1.

Figure 4. Robustness comparison of the standardised method, solid growth and non-standardised inoculum. Biofilm formation (BF/FG, biofilm/final growth ratio) of the four NTHi strains and Pseudomonas aeruginosa $\mathrm{PAO} 1$ positive control (PC) for 8 wells over 13 plates. Error bars depict standard deviation (SD) and with statistical significance $\mathrm{P}<0.05(*)$.

Figure 5. The mean of biofilm production (BF) for four fresh NTHi strains and Pseudomonas aeruginosa PAO1 positive control (PC) over 48 weeks. Error bars depict standard deviation (SD) and with statistical significance

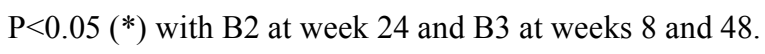


Tables

Table 1. The percent relative deviation \%RSD of the strains for each day. Nonparametric tests found no differences in the \%RSD values between each strain over day 5 or within the strains across days.

\begin{tabular}{|l|l|l|l|l|l|l|l|l|l|l|}
\hline \multirow{2}{*}{ Strain } & \multicolumn{3}{|l}{ Day1 } & \multicolumn{2}{l|}{ Day 2 } & \multicolumn{2}{l|}{ Day 3 } & \multicolumn{2}{l|}{ Day 4 } & \multicolumn{2}{l|}{ Day 5 } \\
\cline { 2 - 11 } & BF & BF/FG & BF & BF/FG & BF & BF/FG & BF & BF/FG & BF & BF/FG \\
\hline A1 & 20.67 & 6.04 & 3.49 & 53.51 & 58.96 & 53.70 & 10.74 & 3.74 & 42.86 & 45.48 \\
\hline A2 & 18.87 & 22.64 & 12.41 & 23.34 & 54.62 & 61.20 & 5.78 & 1.26 & 48.81 & 46.26 \\
\hline A3 & 30.00 & 34.66 & 34.03 & 31.01 & 13.83 & 20.17 & - & - & 9.54 & 11.54 \\
\hline A4 & 14.25 & 1.42 & 31.75 & 32.08 & 9.59 & 10.06 & 10.46 & 12.10 & 80.95 & 85.93 \\
\hline PAO1 & 6.84 & 3.05 & 10.40 & 12.09 & 4.19 & 12.66 & 10.75 & 12.60 & 28.17 & 29.92 \\
\hline
\end{tabular}

Table 2 . Inter-day of \%RSD for the validation of MTP assay methods. Comparison of the three methods (standardised, non-standardised and solid method). Nonparametric tests found no difference between the \%RSD of the standardised method with the other methods.

\begin{tabular}{|l|l|l|l|l|l|l|}
\hline & \multicolumn{2}{|l|}{ Standardised method } & \multicolumn{2}{l|}{ Non-standardised method } & \multicolumn{2}{l|}{ Solid method } \\
\cline { 2 - 8 } & BF & BF/FG & BF & BF/FG & BF & BF/FG \\
\hline A1 & 24.88 & 37.46 & 39.48 & 30.21 & 32.84 & 34.43 \\
\hline A2 & 17.17 & 18.91 & 63.57 & 48.11 & 16.27 & 17.73 \\
\hline A3 & 45.37 & 43.41 & 95.16 & 98.87 & 64.96 & 60.11 \\
\hline A4 & 108.92 & 79.57 & 35.62 & 22.68 & 22.05 & 13.62 \\
\hline PAO1 & 15.59 & 14.42 & 44.67 & 35.24 & 23.96 & 26.83 \\
\hline
\end{tabular}


Figures.

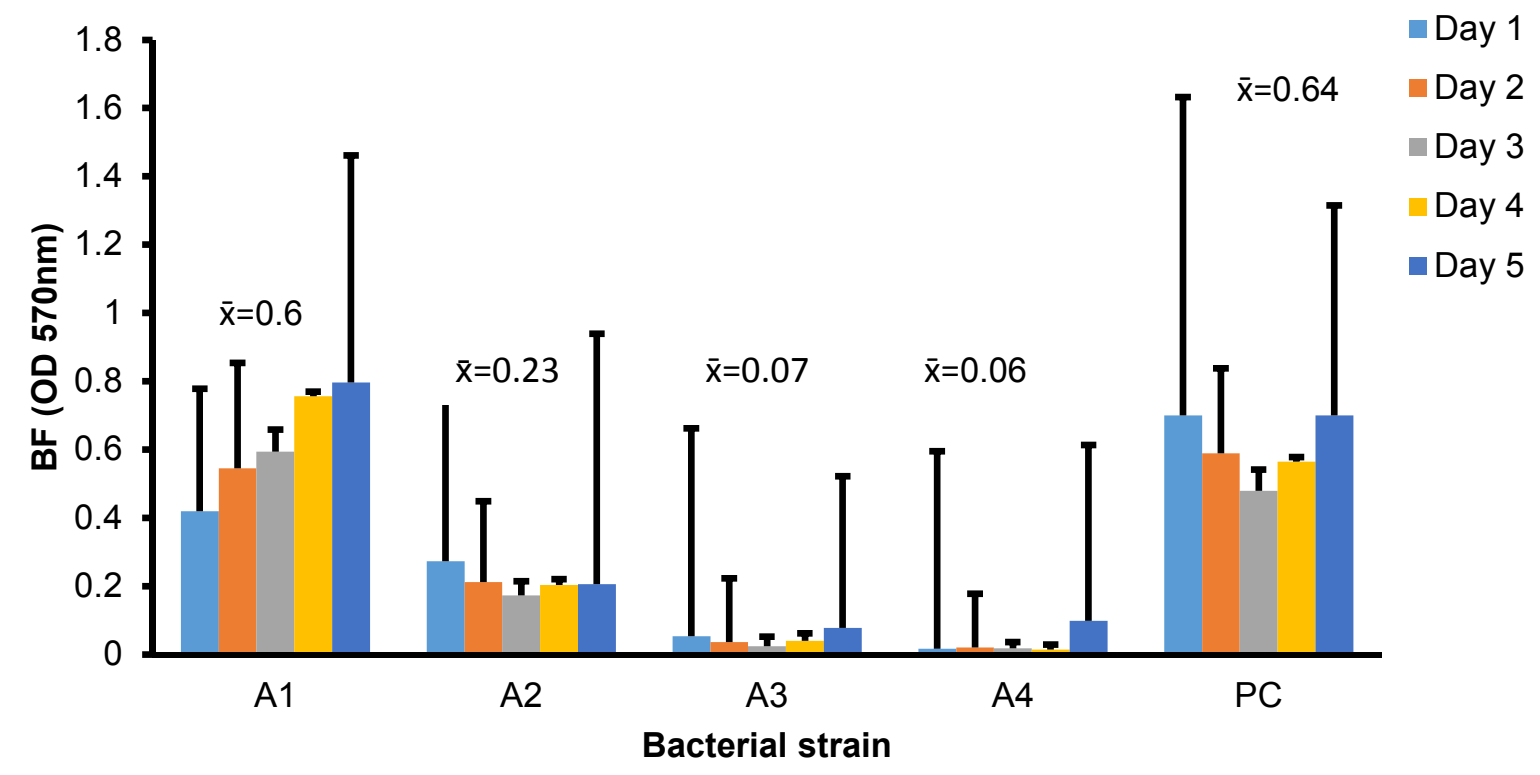

Figure 1. 


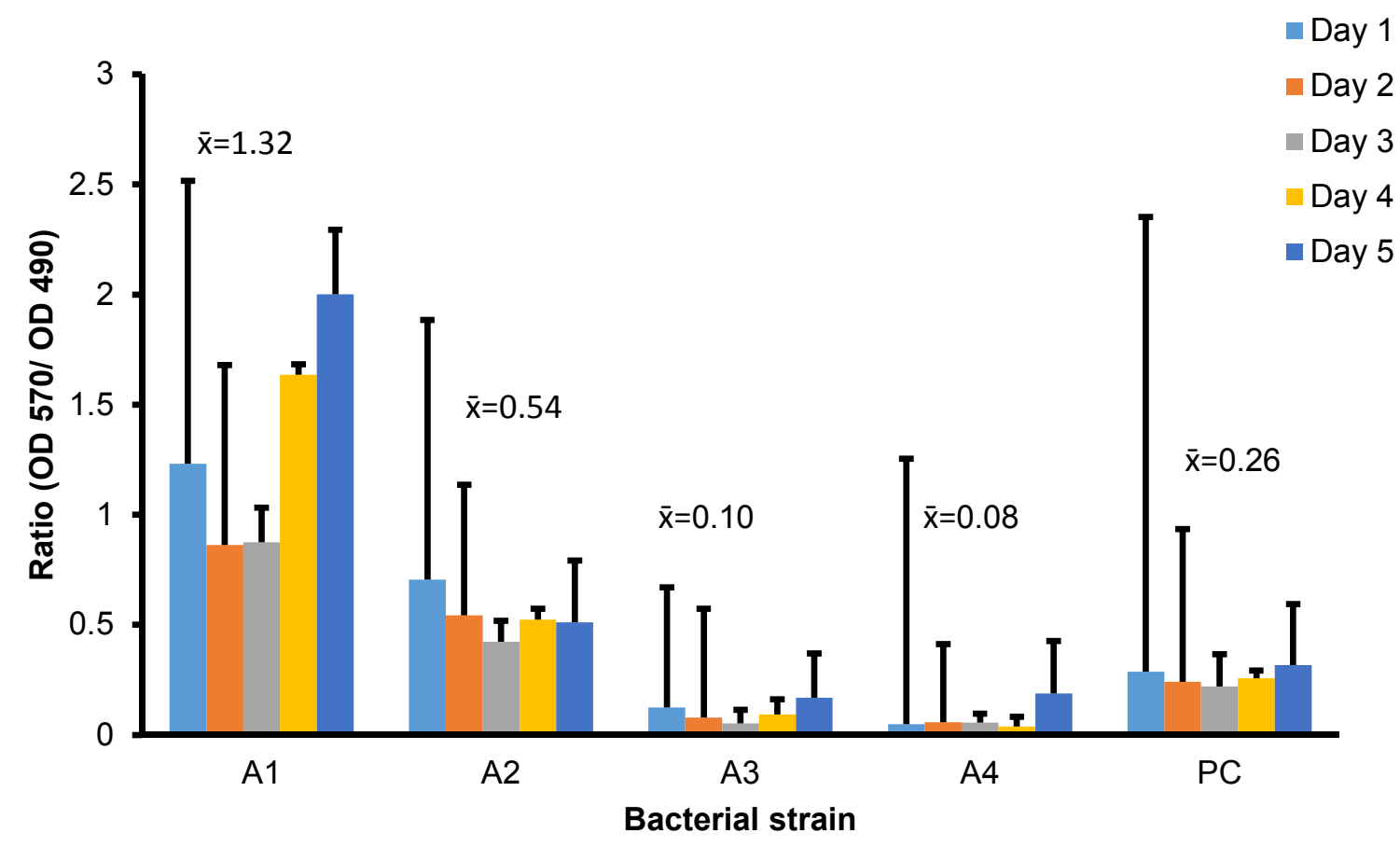

Figure 2. 


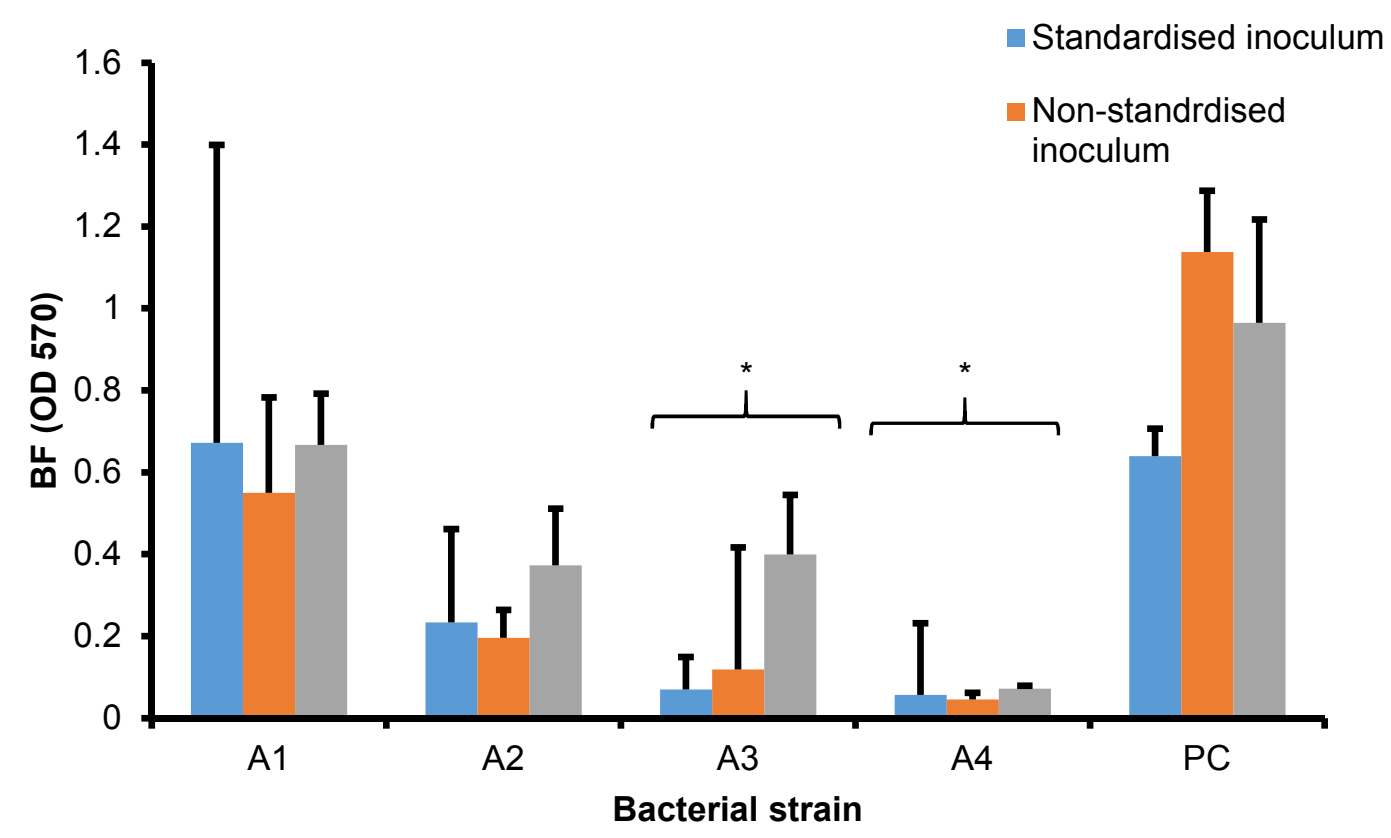

Figure 3 


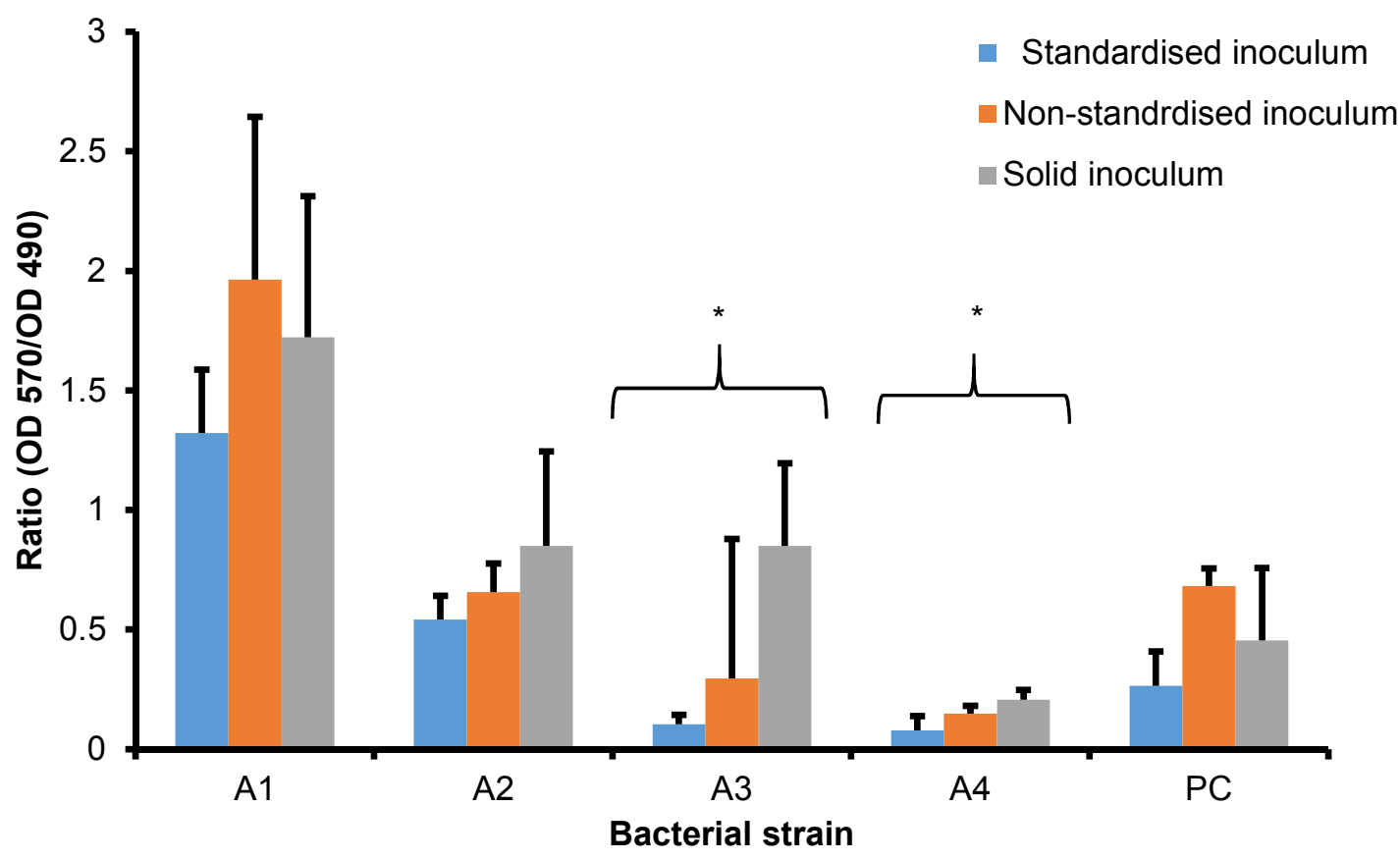

Figure 4 


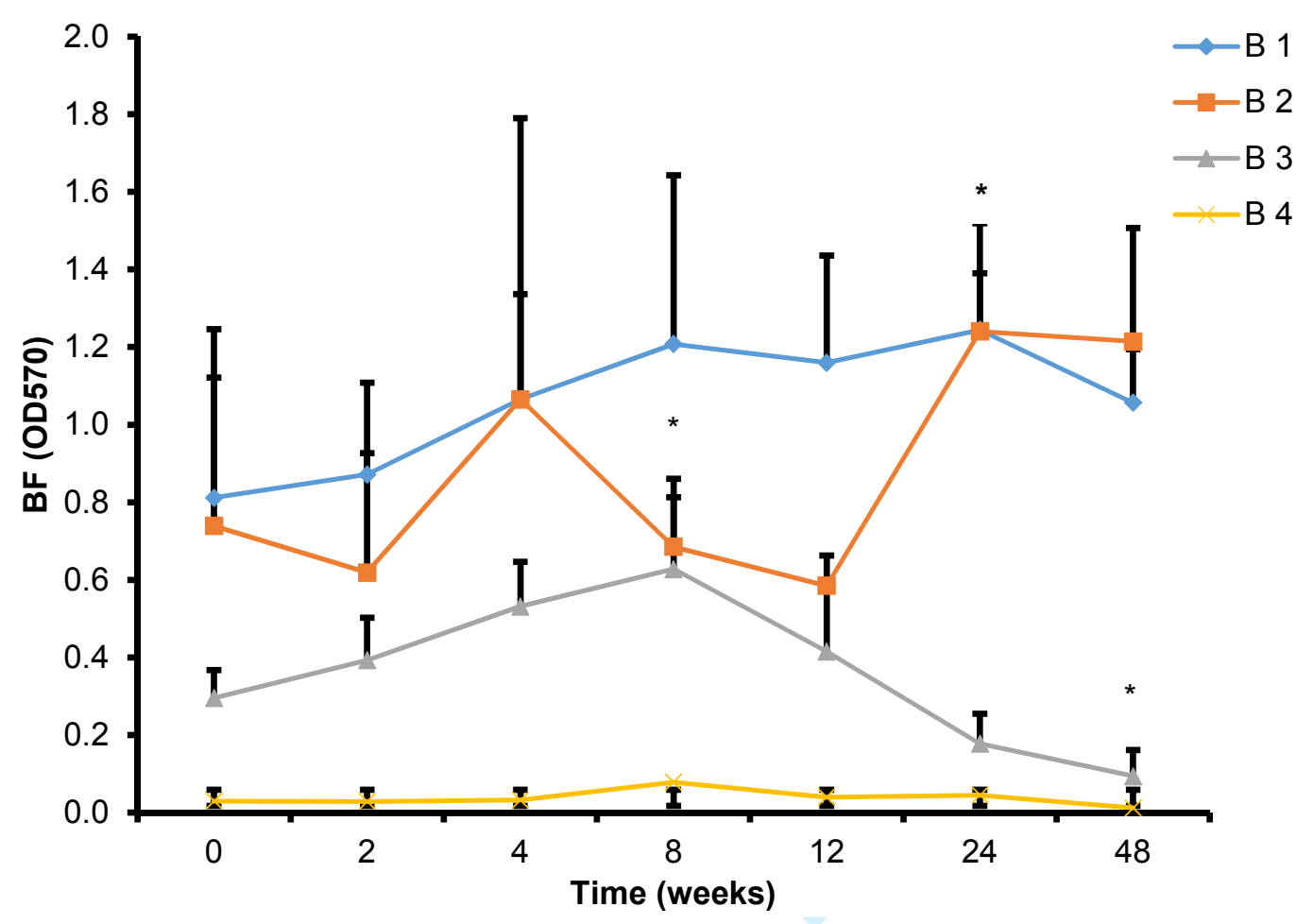

Figure 5. 